\title{
How long does protection last? - In vivo fluorescence confocal laser scanning imaging for the evaluation of the kinetics of a topically applied lotion in an everyday setting
}

\author{
E. Sattler ${ }^{1}$, R. Kästle ${ }^{2}$, M. Arens-Corell ${ }^{3}$ and J. Welzel ${ }^{2}$ \\ ${ }^{1}$ Department of Dermatology and Allergology, Ludwig-Maximilians-University of Munich, Munich, Germany, \\ ${ }^{2}$ Department of Dermatology and Allergology, General Hospital Augsburg, Augsburg, Germany and ${ }^{3}$ Sebapharma GmbH E Co. KG, Boppard, \\ Germany
}

Background: Confocal laser scanning microscopic imaging is well established as a helpful diagnostic tool in dermatology. With a new generation of multi-wave laser confocal microscopes now, in addition to the reflection mode, examinations with fluorescent agents are possible in vivo and ex vivo. Gathering details on the physical, chemical and kinetic features of different fluorophores in different vehicles in healthy skin in vivo will be of interest for therapeutic as well as cosmetic dermatology.

Objectives: Objective of this study was to determine the kinetics of the protective function of a topically applied, fluorescentcontaining lotion in a $24 \mathrm{~h}$ everyday setting in healthy volunteers using fluorescence confocal laser scanning microscopy imaging. Material and methods: Fifteen volunteers received a standardized application of a sodium fluoresceine containing lotion. At several time points of a $24 \mathrm{~h}$ interval confocal laser imaging in the multi-wave mode was performed. Fluorescence was measured by a semiquantitative score and by one based on the intensity of light.

\section{Background}

C ONFOCAL LASER scanning microscopy (CLSM) offers a non-invasive diagnostic view into the upper layers of the skin in almost a histopathologic resolution in vivo in real time. Nowadays, two different imaging modes are available for application in dermatology: the reflectance mode and the fluorescence mode. In the reflectance mode, contrast is achieved by utilizing the inherent refractive index properties of the various cellular microstructures. It is well established as a clinical diagnostic tool for the diagnosis of melanoma and non-melanoma skin
Results: CLSM images in the depths of the stratum corneum and of the upper epidermis of all 15 panelists showed a detectable fluorescence after $30 \mathrm{~min}$ and $4 \mathrm{~h}$ and after $24 \mathrm{~h}$, a partial fluorescence was still visible in 10 of 15 persons. Similar results were achieved using a semiquantitative score and by measuring the intensity of light.

Conclusion: Sodium fluoresceine as a safe and efficient fluorophore for in vivo fluorescence CLSM was able to visualize the kinetics of a topically applied protective lotion and was able to be detected for up to $4 \mathrm{~h}$ in all panelists and even up to $24 \mathrm{~h}$ in two-thirds of the healthy volunteers showing the long duration of the fluorescence on the skin surface, even in an everyday setting.

Key words: confocal laser scanning microscopy - fluorescence - sodium fluoresceine - multiwave - in vivo

cancers (1-11), but also for a wide range of other skin diseases (12-16).

The fluorescence mode relies on the differential distribution of a previously applied exogenous fluorophore to produce contrast. With the new generation of multi-wave confocal laser scanning microscopes a whole variety of options has opened up. In vivo as well as ex vivo devices are available to offer non-invasive real-time images of the skin directly at the patient as well as to scan tissue samples at different laser wave lengths: 445, 658, 785, and $830 \mathrm{~nm}$. The main advantage is that in addition to the reflection mode images, which are possible in wave 
length ranges around these stated laser wave lengths, filter systems offer the possibility to let only the light pass through that is emitted back from a certain fluorescent dye which was excited by that correlating wave length. For the $445 \mathrm{~nm}$ laser, a fitting fluorophore is sodium fluoresceine, whereas for the $658 \mathrm{~nm}$ laser for example methylene blue or for another $785 \mathrm{~nm}$ laser, which can be chosen for CLSM respectively, indocyanin green would be a valid option for fluorescent dyes. Although the use of fluorescence is widely spread among medical diagnostic tools like in fluorescence in situ hybridization (FISH), fluorescent activated cell sorter (FACS), fluorescence microscopy or spectroscopy to name just a few, fluorescence in CLSM was primarily used in the field of scientific dermatologic research, but is finding more and more use in clinical approaches (17). One study compared the advantages and disadvantages of the reflection mode with the fluorescence mode in human skin and showed the benefit of the combination of both imaging modes in vivo (17).

Ex vivo fluorescence CLSM has been shown to be a helpful tool in the evaluation of skin specimens, especially in speeding up and determining tumor margins in Moh's micrographic surgery for basal cell carcinoma(18-21). In vivo fluorescence CLSM was already used for studies on skin surface cellular morphology (22), a pilot study on wound healing (23) and in research concerning penetration into the skin and hair follicles and the barrier function of different dermatologic agents (24-29). Knowledge about the in vivo characteristics of certain fluorophores and their vehicles in healthy skin is needed for the evaluation of their behavior in and on diseased skin in the future.

\section{Material and Methods}

In this prospective experimental study, $24 \mathrm{~h}$ kinetics of the protective function of a sodium fluorescein containing lotion applied topically on the skin surface in an everyday setting were to be examined by CLSM. The test product 'sebamed Lotion' is an oil-in-water lotion with a $\mathrm{pH}$ value of 5.5, containing glycerole, allantoin and chamomille extract as active ingredients (INCI: Aqua, Cetearyl Alcohol, Glycerin, Sorbitol, Paraffinum liquidum, Sodium Lactate, Decyl Oleate, Chamomilla recutita, Lecithin,
Ascorbyl Palmitate, Allantoin, Caprylic/Capric Triglyceride, Sodium Cetearyl Sulfate, Dimethicone, Sodium Citrate, Parfum, Alcohol denat., Methylparaben, Phenoxyethanol, Propylparaben). Fifteen healthy volunteers were recruited after receiving written information on the aim and design of the study and giving consent. Included in the study were only healthy adults between the age of 18 and 60 years with no ongoing skin disease and no known contact sensitization to the ingredients of the applied lotion (including an allergy to fragrances). The lotion was recommended for daily skin care after sporting activities. Therefore, only panelists performing regular sporting were included.

After written informed consent, all panelists received a standardized topical application of the lotion containing sodium fluorescein on the left forearm, which was only allowed to be washed with water before and was otherwise untreated. For this a test field of $3 \times 5 \mathrm{~cm}$ was determined. In this area, $0.02 \mathrm{ml}$ of the fluorecein containing lotion was applied and spread. The concentration of sodium fluoresceine added to the initial $4 \mathrm{~g}$ of lotion equaled $4 \%$. The amount applied was therefore $1.5 \mathrm{mg} / \mathrm{cm}^{2}$ skin. The patterns of fluorescence and its duration were examined by CLSM with a multi-wavelaser at $445 \mathrm{~nm}$ for $30 \mathrm{~min}, 4 \mathrm{~h}$, and $24 \mathrm{~h}$ after application of the lotion. During this time, the panelists were allowed to wash their skin and/ or take a shower as usual without using other skin care or cleansing products. They were also allowed to work and go in for sports.

The study was conducted between April and May 2010 in the Department of Dermatology and Allergology, General Hospital Augsburg.

\section{Initial examination}

Prior to the application of the lotion and the measurements with CLSM, baseline data concerning medical history and skin status were gathered. Documented were: Day of examination, age, gender, status of skin: dry - normal - fatty - mixed skin, known allergies or sensibilizations, atopy yes - no, frequency, kind and duration of sporting activity.

Confocal laser scanning mircroscopy (CLSM)

Imaging of the stratum corneum $(z=5 \mu \mathrm{m})$ and the upper epidermis $(\mathrm{z}=40 \mu \mathrm{m})$ was 
performed using the confocal laser microscope Vivascope 1500 Multiwave (Fa. Mavig, Munich, Germany). Horizontal layers of the skin surface were captured in the fluorescence mode with a laser with a wave length of $445 \mathrm{~nm}$. Fluorescence excitation of the sodium fluoresceine incorporated in the lotion could be selectively imaged with the help of special filters that shut out the reflected light. This way fluorescence gives a bright image, whereas missing fluorescence shows a complete black picture. Single spots of $500 \times 500 \mu \mathrm{m}$ were combined to mosaics (formerly called blocks) of $4 \times 4 \mathrm{~mm}$.

Semiquantitive evaluation of fluorescence was undertaken according to the following score:

Score 3: strong, consistent fluorescence of the entire skin surface

Score 2: strong, but discontinuous fluorescence of the entire skin surface

Score 1: weak fluorescence in the wrinkles

Score 0, 5: punctuated fluorescence

Score 0: no fluorescence.

Images of the CLSM were electronically saved and evaluated morphologically. Exemplary pictures are presented under results.

As the laser power was consistently $4.9 \mathrm{~mW}$ in all mosaic images, their intensity of light could be compared. The intensity of light for each mosaic (block) of $4 \times 4 \mathrm{~mm}$ was measured by Image J. This software program is able to analyze the different levels of a grayscale within the mosaic and then to summarize for each degree of brightness how often this is in the picture, so it measures the brightness of the pixel per area. The possible score lies between 0 and 256.

This way a second more objective and examiner independent score was established depending on the intensity of light (IL-Score):

IL-Score 3: Intensity of light > 25; strong intensity

IL-Score 2: Intensity of light > 12.0-25; moderate intensity

IL-Score 1: Intensity of light $>5.0-12.0$; low intensity

IL-Score 0.5: Intensity of light 4.6-5.0; weak intensity

IL-Score 0: Intensity of light from 0 to $<4.6$; no intensity.

\section{Results}

Of the 15 healthy volunteers, aged 29-57 (median of 46), 2 were men and 13 women. No adverse events and no drop outs were registered.

CLSM

Thirteen of the fifteen panelists showed a consistent and strong fluorescence (score 3) on the entire skin surface of the test area $30 \mathrm{~min}$ after the application of the sodium fluoresceine incorporated in the lotion. Only two of the panelists showed a disconnected pattern of fluorescence, equating to a mean score of 2.87 .

After $4 \mathrm{~h}$ two panelists still showed an unchanged strong and even fluorescence, 12 panelists a strong, but disconnected fluorescence and in one person fluorescence could only be seen within the wrinkles (mean score of 2.07).

After $24 \mathrm{~h}$ one person still showed a strong, yet disconnected fluorescence, six panelists a weak fluorescence within the folds. In three persons, only a punctuated pattern was visible and in five panelists no fluorescence was detectable (mean score of 0.63 ).

Consequently, all 15 panelists showed a detectable fluorescence after $30 \mathrm{~min}$ and $4 \mathrm{~h}$ and after $24 \mathrm{~h}$ a rest of fluorescence was still visible in 10 out of 15 persons (Figs 1 and 2).

As the evaluation of the intensity of light is not dependent on the pattern of partially disconnected fluorescence, but on the brightness of the measured fluorescence, slight differences were seen in the the IL-score compared to the semiquantitative score (Fig. 3).

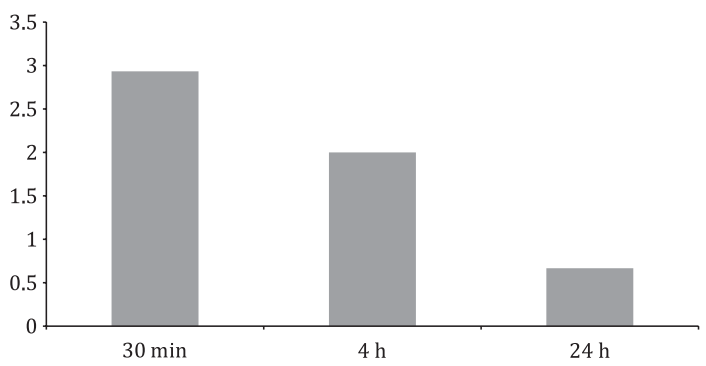

Fig. 1. Mean semiquantitative score of fluorescence as a function of time. 


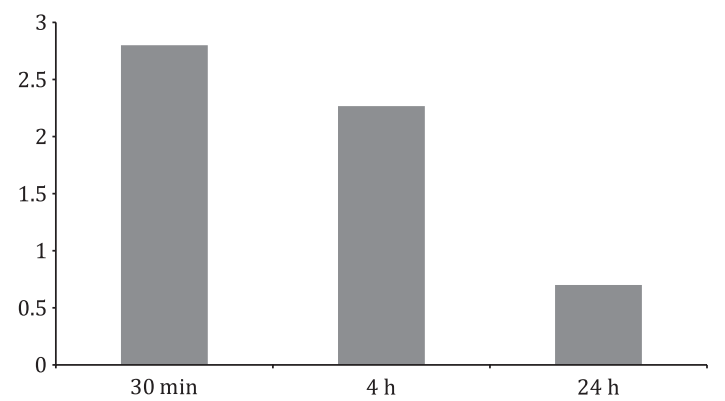

Fig. 2. Mean score of intensity of light (IL-score) as a function of time.

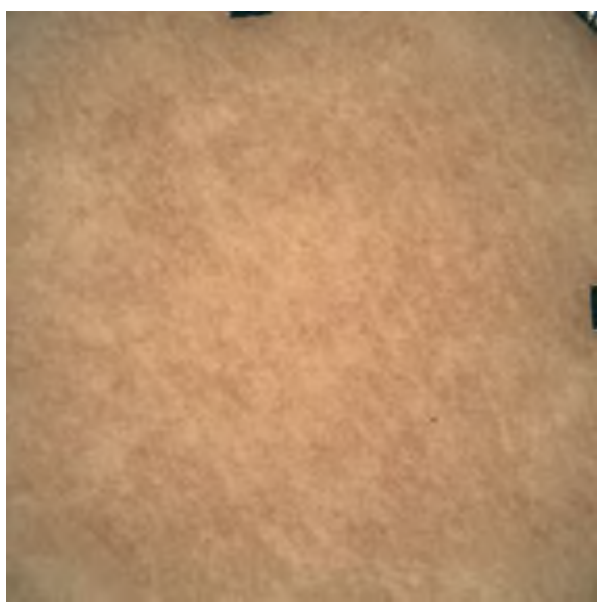

Fig. 3. Macroscopic picture of the skin. Fluorescein causes only very little yellow discoloration which is bearly seen on the skin.

Evaluation of the Intensity of light score showed the following results:

After $30 \mathrm{~min}, 12$ out of 15 panelists had a strong intensity of light (IL-Score of 3) and three persons displayed a light intensity with a

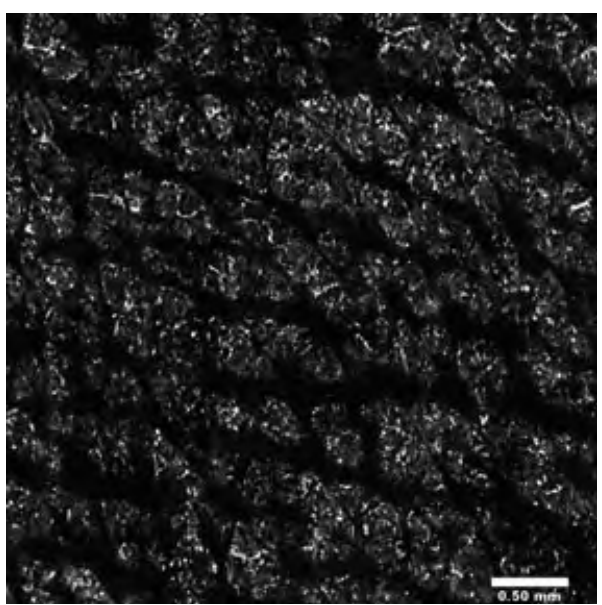

Fig. 4. Thirty minutes after application, superficial mosaic (formerly block) of the stratum corneum $(z=10 \mu \mathrm{m})$. The entire skin surface shows a strong and even fluorescence.

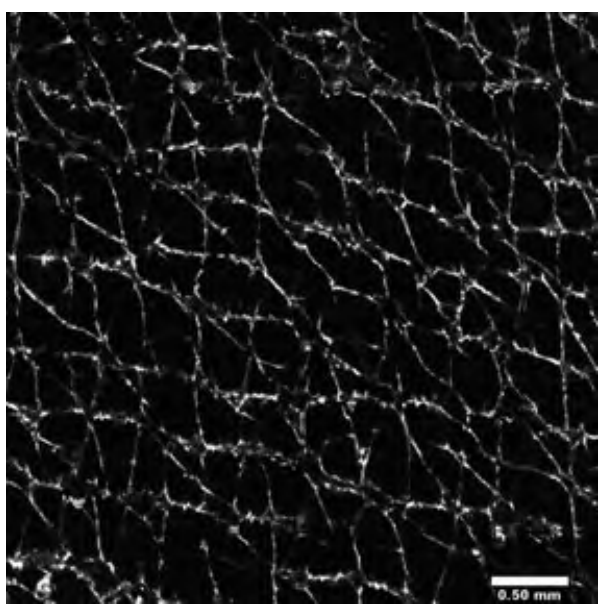

Fig. 5. Thirty minutes after application, mosaic (block) of the upper epidermis. The folds of the hearbearing skin are filled with fluorescent lotion $(z=40 \mu \mathrm{m})$.

score of 2 (Figs 4 and 5). Four hours after the application, five panelists still showed a strong intensity of light, nine a moderate intensity (IL-score of 2) and one only a low intensity (IL-score of 1) (Figs 6 and 7). At the last measuring time point, $24 \mathrm{~h}$ after application, one person still showed a moderate intensity (ILscore of 2), seven panelists still had a low intensity (IL-score of 1) and two panelists showed a weak intensity of light (equaling an IL-score of 0.5) (Fig. 8). In five persons, no light intensity was detectable after $24 \mathrm{~h}$.

Examplary Fluorescence images by CLSM, at each time point two images were taken from the height of the stratum corneum and of the upper epidermis (folds):

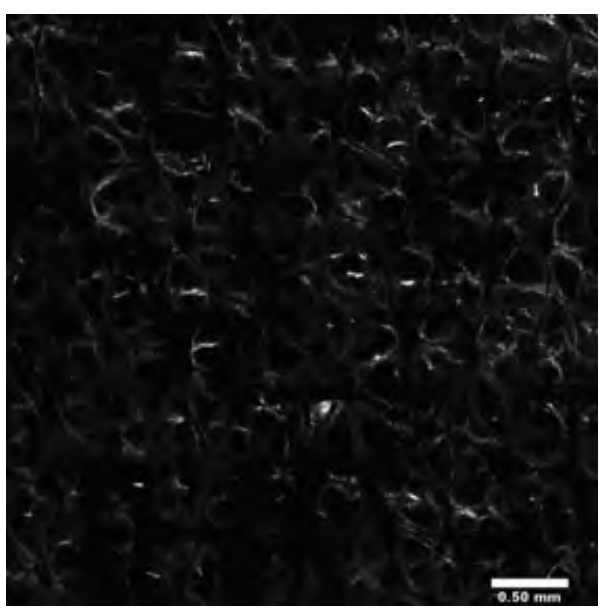

Fig. 6. Four hours after application, only an inconsistent pattern of fluorescence is still visible on the skin surface $(z=10 \mu \mathrm{m})$. 


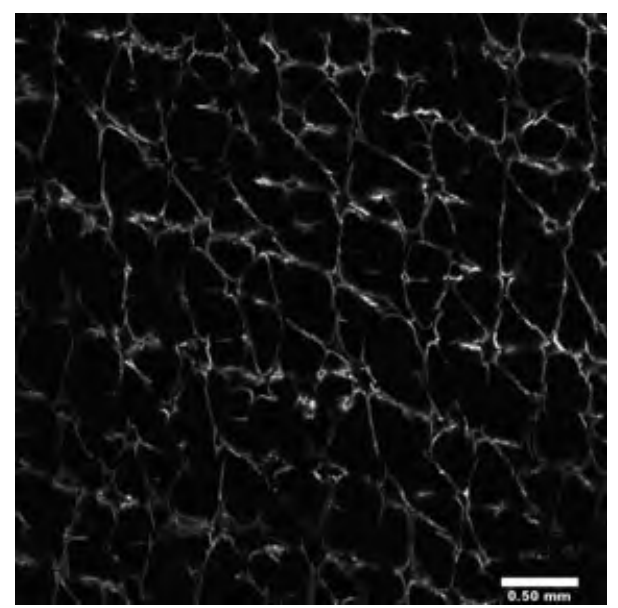

Fig. 7. Four hours after application, still a strong fluorescence within the wrinkles is detectable, but already partially disconnected $(z=40 \mu \mathrm{m})$.

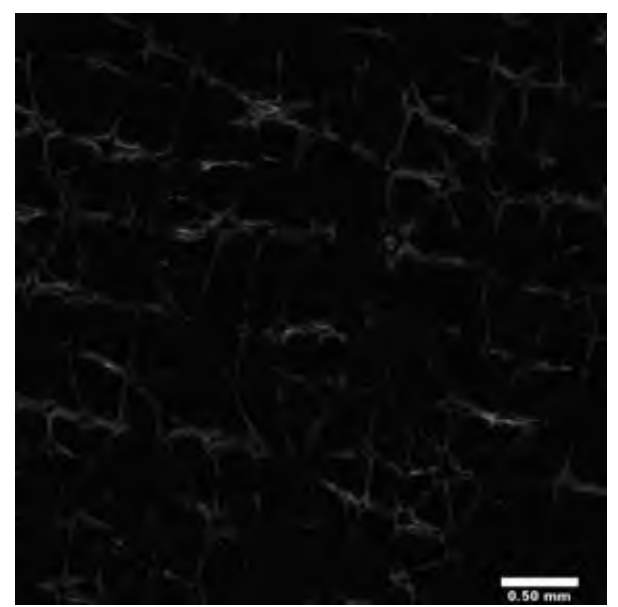

Fig. 8. Twenty-four hours after application, the picture of the skin surface shows no fluorescence left (not shown), yet within the folds still a weak, but interrupted fluorescence is visible $(z=40 \mu \mathrm{m})$.

\section{Discussion}

Exploring a new field of diagnostic agents, in this case of fluorophores suitable for in vivo fluorescence CLSM, of course the first concern has to be directed towards safety. For this reason, in this study, sodium fluoresceine was chosen for the in vivo application on the skin surface. It is a widely used fluorophore not only in other medical applications in health care especially ophthalmology and optometry but also in everyday use like analyzing river systems, finding water leaks or even as food additive dye, that is called D \& C Yellow no. 8 (approved of by the Food and Drug Administration). Yet, topical, oral, and intravenous use of fluoresceine has been reported to be able to cause adverse reactions ranging from nausea, vomiting, hives, acute hypotension to very rare cases of anaphylaxis(30-34). In a study of 6524 patients with retinoic angiography with fluoresceine, adverse experiences were seen in $7.5 \%$, severe reactions occurred in $0.27 \%$ of patients, but only in 2 of the 6524 patients a resuscitation $(0.03 \%)$ was necessary(35). In our study none of these described adverse events were seen. Still like with any other agent health care provider who use fluoresceine should be prepared and able to perform emergency management with any ensuing anaphylaxis.

Sodium fluoresceine is the water-soluble sodium salt of fluoresceine and is also called uranine. It is a yellow, under UV- and daylight green fluorescent dye. Fluorescence is the emission of light by a substance that has absorbed light or other electromagnetic radiation of a different wavelength. In most cases, the emitted light has a longer wavelength and therefore lower energy than the absorbed radiation. As fluoresceine has its absorption maximum at $485 \mathrm{~nm}$ (pH9) and its emission maximum at $514 \mathrm{~nm}$ it was excited by the $445 \mathrm{~nm}$ laser of the Vivascope 1500 Multilaser (Fa. Mavig) and fluorescence was distinguished from reflected light using a special filter system. As presented, the fluorescence could very well be detected by CLSM in vivo at the height of the stratum corneum as well as in the folds of the upper epidermis. As the sodium fluorescein was contained and applied topically in a lotion it was used to visualize the kinetics of the applied lotion on and in the skin. The main goal was to see how long the protective function of the lotion can be kept under normal circumstances. In both scores, the semiquantitative as well as in the more objective intensity of light score, fluorescence was strong at $30 \mathrm{~min}$ after topical application as well as moderate after $4 \mathrm{~h}$ in almost all panelists and lasted up to $24 \mathrm{~h}$ in two-thirds of the 15 volunteers, even though they were allowed and supposed to follow their typical individual everyday routine to allow for a realistic everyday setting. This shows that even in normal everyday life the protective function of the lotion lasted relatively long. The observed eventual abatement of fluorescence can be explained in three ways:

(1) First of all fluorophores like sodium fluorescein are subject to photobleaching. Photo- 
bleaching is the photochemical destruction of a fluorophore and it may complicate the observation of fluorescent molecules over time, as they will eventually be destroyed by the light exposure necessary to stimulate them into fluorescing. Loss of activity caused by photobleaching can be controlled by increasing the concentration of the fluorophore, by reducing the intensity or time-span of light exposure, by reducing the frequency and thus the photon energy of the input light or by employing more robust fluorophores that are less prone to bleaching. Depending on the material, dyes can produce different photon numbers and therefore have different lifetimes, ranging from 0.1 to $1 \mathrm{~s}$ (s) with green fluorescent protein, over 1-10 s for typical organic dyes to more than $1000 \mathrm{~s}$ for very stable fluorphores like CdSe/ZnS Quantum dot. In our setting, due to the fixed wave lengths of the laser device and the matching special filters the only variable to be optimized could be the concentration of the sodium fluorescein. As we already found a fairly long fluorescence, the used concentration seems to be adequate. The question, if a lower concentration would also be sufficient, should be addressed in further studies.

(2) The fluorophore was not applied alone topically, but as an ingredient of the tested lotion. The lotion and with it the contained fluorophore may have been adsorbed and may have penetrated into the skin and/or may have been metabolized. On the other side, fluorescein is water soluble and hydrophilic. Therefore, it is emulsified in the water part of the lotion. It might be that the lipid part of the lotion is adsorbed and penetrated into the skin and that the water part together with the fluorophore stays on the surface. If the water evaporates over time, only the fluorescent dye and not the whole emulsion remains on the skin surface.

(3) Last and probably not the least, of course parts of the fluorescein containing lotion would have been rubbed off by the panelists in intentionally doing their regular $24 \mathrm{~h}$ routine including washing the skin, going to work and even sports. This was intended to read out how long protection by the lotion is really offered not in a fancy study design, but in everyday life.
In addition, our results could not only show that the lotion remained relatively long on the skin possibly fulfilling its protective effect and the relatively slow fading over time, but also add some information on the distribution of the lotion on and in the skin. As shown in the exemplary pictures, a big amount of the lotion goes into the folds of the skin and this is also where it remains for the longest time as shown by the $24 \mathrm{~h}$ results.

A similar study used the lipophilic dye curcumin incorporated in an oil-in-water microemulsion and in an amphiphilic cream both applied topically and measured the strength and the distribution of fluorescence in the stratum corneum using CLSM(36). For removal of the stratum corneum, the method of tape stripping was chosen and for the investigation of the penetration of the hair follicles cyanoacrylate surface biopisies were performed. Although in the case of the amphiphilic cream little penetration in deeper layers of the Stratum corneum took place and the main amount of the dye stayed on the skin surface, with the mircoemulsion a smaller amount of curcumin remained on the skin surface and penetration of the stratum corneum was much deeper with decreasing amounts still detectable up to a relative stratum corneum depth of about $80 \%$. Concerning follicular penetration, penetration of the complete follicular infundibula were seen with the microemulsion, whereas with the fluorophore containing cream, signals were only gathered from the follicular orifices(36). In another study, the same group could show that barrier-enhancing formulations were able to prevent the penetration of Pollen allergens into the hair follicles (37). The extent of the preventive effect depended on the formulation, but the reduction of penetration was significantly higher in all formulations compared with untreated skin, proving a similar effect like the protective function of the lotion seen in our study. A pilot study by Leeson et al. could show a strong variation in skin surface cellular morphology using fluoresceine aided in vivo fluorescence CLSM(22). They found not only a correlation between dryness of the skin and the intensity of fluorescence, but could demonstrate in a number of clinical studies that the cellular morphology of the corneocytes at the skin surface correlates well with the overall condition of skin and that it is shown to be directly affected by treatment that influence 
the efficiency of the process of desquamation. In another study, fluorescence CLSM was tested in a clinical setting and allowed monitoring of the effects of a topically applied wound ointment on the kinetics of wound healing noninvasively and over time (23). Another clinical question was addressed by a group that used fluorescence CLSM for in vivo imaging of epidermal reactions to two experimental irritants. In this experimental contact dermatitis model, the fluorophore administered topically and intradermally was also sodium fluoresceine with a $488 \mathrm{~nm}$ laser. Disturbed and widened cell borders, swelling of keratinocytes, and induction of a parakeratotic shift could be visualized by fluorescence CLSM(38).
We feel that in vivo fluorescence CLSM offers an interesting approach valuable in pharmaceutical or cosmetical research as well as in all skin diseases altering desquamation and epidermal structures and should be investigated in future studies. Further questions to be addressed should be the right vehicle for the best fluorophore and the optimum laser wave length (other substances that can function as fluorophores are e.g. rhodamine B, Acridine Orange, green fluorescent protein, curcumin with absorption maxima at different laser wave lengths (39)), and further details on penetration and other skin physiologic characteristics to optimize the informative value of fluorescence CLSM in vivo.

\section{References}

1. Gonzalez S. Confocal reflectance microscopy in dermatology: promise and reality of non-invasive diagnosis and monitoring. Actas Dermosifiliogr 2009; 100(Suppl. 2): 59-69.

2. Gonzalez S, Swindells K, Rajadhyaksha $M$ et al. Changing paradigms in dermatology: confocal microscopy in clinical and surgical dermatology. Clin Dermatol 2003; 21: 359-369.

3. Pellacani G, Longo C, Malvehy J et al. In vivo confocal microscopic and histopathologic correlations of dermoscopic features in 202 melanocytic lesions. Arch Dermatol 2008; 144: 1597-1608.

4. Pellacani G, Scope A, Ferrari B et al. New insights into nevogenesis: in vivo characterization and follow-up of melanocytic nevi by reflectance confocal microscopy. J Am Acad Dermatol 2009; 61: 1001 $-1013$.

5. Rajadhyaksha M, Gonzalez S, Zavislan JM et al. In vivo confocal scanning laser microscopy of human skin II: advances in instrumentation and comparison with histology. J Invest Dermatol 1999; 113: 293-303.

6. Rajadhyaksha M, Menaker G, Flotte $\mathrm{T}$ et al. Confocal examination of nonmelanoma cancers in thick skin excisions to potentially guide mohs micrographic surgery without frozen histopathology. J Invest Dermatol 2001; 117: 11371143.

7. Selkin B, Rajadhyaksha M, Gonzalez $S$ et al. In vivo confocal micros- copy in dermatology. Dermatol Clin 2001; 19: 369-377.

8. Ulrich M, Gonzalez S, Lange-Asschenfeldt $B$ et al. Non-invasive diagnosis and monitoring of actinic cheilitis with reflectance confocal microscopy. J Eur Acad Dermatol Venereol 2011; 25: 276-284.

9. Ulrich M, Krueger-Corcoran D, Roewert-Huber J et al. Reflectance confocal microscopy for noninvasive monitoring of therapy and detection of subclinical actinic keratoses. Dermatology 2010; 220: 15-24.

10. Ulrich M, Maltusch A, Rius-Diaz F et al. Clinical applicability of in vivo reflectance confocal microscopy for the diagnosis of actinic keratoses. Dermatol Surg 2008; 34: 610-619.

11. Ulrich M, Maltusch A, Rowert-Huber $\mathrm{J}$ et al. Actinic keratoses: noninvasive diagnosis for field cancerisation. Br J Dermatol 2007; 156 (Suppl. 3): 13-17.

12. Astner S, Gonzalez S, Cuevas J et al. Preliminary evaluation of benign vascular lesions using in vivo reflectance confocal microscopy. Dermatol Surg 2010; 36: 1099-1110.

13. Gonzalez S, Gilaberte-Calzada Y. In vivo reflectance-mode confocal microscopy in clinical dermatology and cosmetology. Int J Cosmet Sci 2008; 30: 1-17.

14. Gonzalez S, Rajadhyaksha M, Rubinstein G et al. Characterization of psoriasis in vivo by reflectance confocal microscopy. J Med 1999; 30: 337-356.

15. Astner S, Gonzalez S, Gonzalez E. Noninvasive evaluation of allergic and irritant contact dermatitis by in vivo reflectance confocal microscopy. Dermatitis 2006; 17: 182-191.

16. Astner S, Ulrich M. Confocal laser scanning microscopy. Hautarzt 2010; 61: 421-428.

17. Meyer LE, Otberg N, Sterry W et al. In vivo confocal scanning laser microscopy: comparison of the reflectance and fluorescence mode by imaging human skin. J Biomed Opt 2006; 11: 044012.

18. Ericson MB, Simonsson C, Guldbrand $S$ et al. Two-photon laserscanning fluorescence microscopy applied for studies of human skin. J Biophotonics 2008; 1: 320-330.

19. Gareau DS, Karen JK, Dusza SW et al. Sensitivity and specificity for detecting basal cell carcinomas in Mohs excisions with confocal fluorescence mosaicing microscopy. J Biomed Opt 2009; 14: 034012.

20. Karen JK, Gareau DS, Dusza SW et al. Detection of basal cell carcinomas in Mohs excisions with fluorescence confocal mosaicing microscopy. Br J Dermatol 2009; 160: 1242-1250.

21. Paoli J, Smedh M, Wennberg AM et al. Multiphoton laser scanning microscopy on non-melanoma skin cancer: morphologic features for future non-invasive diagnostics. J Invest Dermatol 2008; 128: 1248 1255.

22. Leeson DT, Lynn Meyers C, Subramanyan K. In vivo confocal fluorescence imaging of skin surface cellular morphology: a pilot study of its potential as a clinical tool in skin research. Int $\mathrm{J}$ Cosmet Sci 2006; 28: 9-20.

23. Lange-Asschenfeldt B, Alborova A, Kruger-Corcoran D et al. Effects of 
a topically applied wound ointment on epidermal wound healing studied by in vivo fluorescence laser scanning microscopy analysis. J Biomed Opt 2009; 14: 054001.

24. Lademann J, Ilgevicius A, Zurbau $\mathrm{O}$ et al. Penetration studies of topically applied substances: Optical determination of the amount of stratum corneum removed by tape stripping. J Biomed Opt 2006; 11: 054026.

25. Lademann J, Knorr F, Richter $\mathrm{H}$ et al. Hair follicles-an efficient storage and penetration pathway for topically applied substances. Summary of recent results obtained at the Center of Experimental and Applied Cutaneous Physiology, Charite -Universitatsmedizin Berlin, Germany. Skin Pharmacol Physiol 2008; 21: 150-155.

26. Lademann J, Patzelt A, Richter $\mathrm{H}$ et al. Comparison of two in vitro models for the analysis of follicular penetration and its prevention by barrier emulsions. Eur J Pharm Biopharm 2009; 72: 600-604.

27. Lademann J, Richter H, Golz K et al. Influence of microparticles on the homogeneity of distribution of topically applied substances. Skin Pharmacol Physiol 2008; 21: 274-282.

28. Lademann J, Richter H, Meinke M et al. Which skin model is the most appropriate for the investigation of topically applied substances into the hair follicles? Skin Pharmacol Physiol 2010; 23: 47-52.

29. Lademann J, Richter H, Schanzer S et al. Penetration and storage of particles in human skin: perspectives and safety aspects. Eur J Pharm Biopharm 2011; 77: 465468.

30. Bearelly S, Rao S, Fekrat S. Anaphylaxis following intravenous fluorescein angiography in a vitreoretinal clinic: report of 4 cases. Can J Ophthalmol 2009; 44: 444-445.

31. Hitosugi M, Omura K, Yokoyama $\mathrm{T}$ et al. An autopsy case of fatal anaphylactic shock following fluorescein angiography: a case report. Med Sci Law 2004; 44: 264 -265 .

32. Kalogeromitros DC, Makris MP, Aggelides XS et al. Allergy skin testing in predicting adverse reactions to fluorescein: a prospective clinical study. Acta Ophthalmol 2009; 89: 480-483.

33. Kwan AS, Barry C, McAllister IL et al. Fluorescein angiography and adverse drug reactions revisited: the lions eye experience. Clin Experiment Ophthalmol 2006; 34: 33-38.

34. Shahid H, Salmon JF. Anaphylactic response to topical fluorescein $2 \%$ eye drops: a case report. J Med Case Reports 2010; 4: 27.

35. Lepri A, Salvini R, Rizzo L et al. Accident during retinal fluorescein angiography. Minerva Anestesiol 1997; 63: 133-140.
36. Teichmann A, Heuschkel S, Jacobi $\mathrm{U}$ et al. Comparison of stratum corneum penetration and localization of a lipophilic model drug applied in an $\mathrm{o} / \mathrm{w}$ microemulsion and an amphiphilic cream. Eur J Pharm Biopharm 2007; 67: 699-706.

37. Meinke MC, Patzelt A, Richter H et al. Prevention of follicular penetration: barrier-enhancing formulations against the penetration of pollen allergens into hair follicles. Skin Pharmacol Physiol 2011; 24: 144-150.

38. Suihko C, Serup J. Fluorescence confocal laser scanning microscopy for in vivo imaging of epidermal reactions to two experimental irritants. Skin Res Technol 2008; 14: 498-503.

39. Suihko C, Swindle LD, Thomas SG et al. Fluorescence fibre-optic confocal microscopy of skin in vivo: microscope and fluorophores. Skin Res Technol 2005; 11: 254-267.

Address:

Prof. Dr med. J. Welzel

Head of the Department of Dermatology

and Allergology

General Hospital Augsburg

Sauerbruchstraße 6

86179 Augsburg

Germany

Tel: +49 821-400-7401

Fax: +49 821-400-17-7401

e-mail: julia.welzel@klinikum-augsburg.de 